\title{
Research on material selection of force- sensitive element for high-frequency dynamic piezoelectric pressure sensor
}

\author{
Sikang Cai, Guicong Wang*, Yingjun Li, and Xiaoqi Yang \\ School of Mechanical Engineering, University of Jinan, Jinan, China
}

\begin{abstract}
The high-frequency dynamic piezoelectric pressure sensor has the advantages of simple structure, long service life, high natural frequency, excellent signal-to-noise ratio and great sensitivity. It is appropriate for measuring high dynamic, dynamic or quasi-static pressure changes and pressure fluctuations. And this kind of sensor is widely utilized in the shock wave testing. The force-sensitive element is one of the main factors affecting the static and dynamic performance of piezoelectric pressure sensors. Basing on the piezoelectric equation and coupling effect between mechanics and electricity, in this paper, the finite element model of the high-frequency dynamic piezoelectric pressure sensor is established. The influences of the force-sensing element on the sensitivity of the sensor are analysed. Referential suggestions for choosing force-sensitive element of high-frequency dynamic piezoelectric pressure sensor are provided.
\end{abstract}

Keywords: Pressure sensor, Piezoelectric ceramics, High-frequency dynamic measure, Charge sensitivity.

\section{Introduction}

In occasions such as shock wave measurement and aerodynamic tests, pressure is a kind of transient signal changing swiftly. And the resolution is needed to reach millisecond or even microsecond level. At the same time, noise interferences are introduced by mechanical vibration or other factors, which requires the pressure sensor selected to have a wide range of response frequency and high natural frequency. High-frequency dynamic piezoelectric pressure sensor has the advantages of high natural frequency, excellent signal-to-noise ratio and great sensitivity. It is appropriate for measuring the signals and is widely employed in occasions such as explosion, internal combustion engine system and aerospace [1].

Piezoelectric material is one of the main factors affecting the measuring performance of the sensor. Nowadays, the piezoelectric materials widely used include piezoelectric quartz, ceramics and film. Piezoelectric quartz is an anisotropic piezoelectric single crystal material with high insulation resistance. But quartz has low piezoelectric coefficient and complicated manufacturing process [2]. Piezoelectric ceramic is piezoelectric

\footnotetext{
* Corresponding author: me wanggc@,ujn.edu.cn
} 
polycrystalline material. Due to its high piezoelectric coefficient, PZT series ceramic are widely utilized. Y.J. Li [3-4] employed PZT-5 as the force-sensitive element to manufacture a piezoelectric pressure sensor with high sensitivity, good dynamic characteristic and acceleration compensation module. Piezoelectric film has the advantages of flexibility and high piezoelectric constant. However, its charge leaks rapidly. The measurement of high-frequency pressure requires the sensor to have a wide response frequency band, high natural frequency and rapid response speed. Basing on the characteristics of the above materials, ceramic is more suitable. However, there are many kinds of ceramics. How to choose appropriate one is a key technical problem that needs to be solved urgently in the process of designing and manufacturing high-frequency dynamic piezoelectric pressure sensors.

\section{Sensor structure modeling}

The piezoelectric pressure sensor is mainly composed of diaphragm, piezoelectric ceramic crystal group, sensor shell, plastic parts and others. Figure 1 is the structure diagram of the sensor. The diaphragm bears the external pressure and transmits this to the crystal group. After the crystal group bears the pressure, it generates electric charge. The charge signal is led out by the copper electrode and the wire through the plastic column. The upper surface of the upper ceramic chip and the lower surface of the lower ceramic ring are grounded through the sensor shell. The plastic ring and the plastic column can reduce the interference of vibration, and the lower shell and the upper shell play a role of supporting and protecting force sensor.

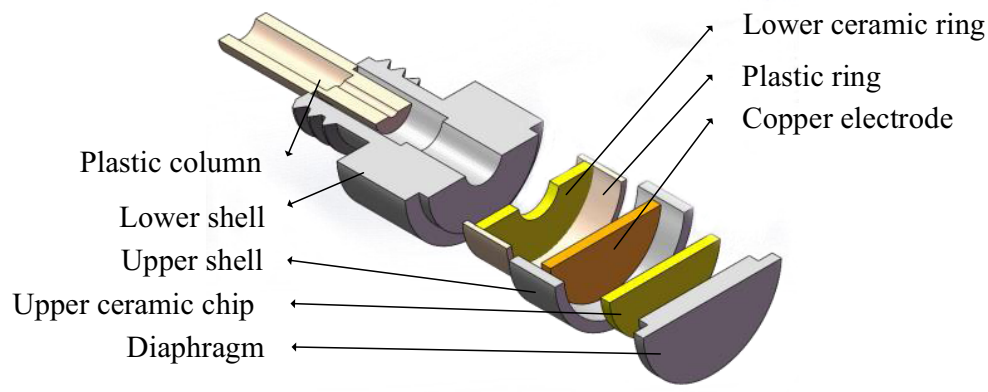

Fig. 1. Sensor structure diagram.

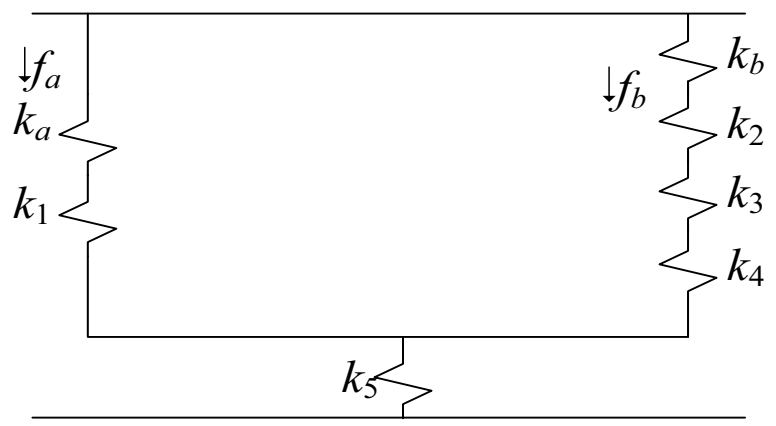

Fig. 2. Equivalent static model of sensor.

Basing on the mechanism modeling method, the equivalent static model of the sensor is established as figure 2 shows. 
where, $k_{a}$ is the equivalent stiffness acting on the contact part of diaphragm and shell, $k_{b}$ is the equivalent stiffness acting on the contact part of diaphragm and crystal group, $k_{1}$ is the equivalent stiffness of upper shell, $k_{2}$ is the equivalent stiffness of upper ceramic plate, $k_{3}$ is the equivalent stiffness of copper electrode, $k_{4}$ is the equivalent stiffness of lower ceramic ring, $f_{a}$ is the load component distributed through shell, $f_{b}$ is the load component distributed through crystal group.

According to fig.2, the voltage sensitivity $S_{F U}$ of the sensor can be obtained as shown in equation. (1).

$$
S_{F U}=\frac{k_{x} d_{i j} n h}{\left(k_{1}+k_{k}\right) \varepsilon_{0} \varepsilon_{r} S}
$$

where, $k_{x}$ is the equivalent stiffness of the entire crystal group, $n$ is the number of wafers, $d_{i j}$ is the piezoelectric constant of the wafer, $h$ is the thickness of the wafer, $\varepsilon_{0}$ is the vacuum permittivity, and $\varepsilon_{r}$ is the relative permittivity of the force sensor, $S$ is the area of the wafer.

Table 1. Comparison of piezoelectric ceramic material parameters.

\begin{tabular}{lcccccccc}
\hline & \multicolumn{8}{c}{ Materials } \\
\cline { 2 - 9 } Parameters & PZT-33 & PZT-4 & PZT-41 & PZT-42 & PZT-51 & PZT-5A & PZT-5H & PZT-81 \\
\hline$d_{33}(\mathrm{pC} / \mathrm{N})$ & 450 & 289 & 250 & 290 & 550 & 374 & 593 & 250 \\
$k_{15}$ & 0.70 & 0.71 & 0.66 & 0.67 & 0.74 & 0.71 & 0.675 & 0.66 \\
$\varepsilon_{r 3}^{T}$ & 1900 & 1300 & 1050 & 1275 & 2400 & 1700 & 3400 & 1100 \\
$\rho\left(\mathrm{kg} / \mathrm{m}^{3}\right)$ & 7750 & 7500 & 7700 & 7650 & 7650 & 7750 & 7500 & 7650 \\
$Y_{11}^{E}\left(10^{9} \mathrm{~N} / \mathrm{m}^{2}\right)$ & 63.29 & 81.30 & 83.33 & 86.96 & 66.67 & 60.98 & 60.61 & 86.96 \\
$\sigma$ & 0.41 & 0.35 & 0.31 & 0.31 & 0.34 & 0.36 & 0.28 & 0.28 \\
$T_{c}\left({ }^{\circ} \mathrm{C}\right)$ & 360 & 328 & 310 & 300 & 290 & 365 & 193 & 310 \\
\hline
\end{tabular}

The main parameters that affect the performance of piezoelectric ceramic materials are piezoelectric constant $d_{33}$, electromechanical coupling coefficient $k_{15}$, dielectric coefficient $\varepsilon_{r 3}^{T}$, density $\rho$, elastic modulus $Y_{11}^{E}$, Poisson's ratio $\sigma$, and Curie point $T_{c}$ [5]. And then, PZT-33, PZT-4, PZT-5A, etc. are selected. The parameters are shown in the table 1.

The theoretical values of voltage sensitivity of various piezoelectric ceramic materials can be calculated by equation (1) as shown in the table 2 .

Table 2. Theoretical voltage sensitivity of piezoelectric ceramics.

\begin{tabular}{lcccccccc}
\hline & \multicolumn{10}{c}{ Materials } \\
\cline { 2 - 9 } Parameter & $\begin{array}{c}\text { PZT- } \\
33\end{array}$ & PZT-4 & $\begin{array}{c}\text { PZT- } \\
41\end{array}$ & $\begin{array}{c}\text { PZT- } \\
42\end{array}$ & $\begin{array}{c}\text { PZT- } \\
51\end{array}$ & $\begin{array}{c}\text { PZT- } \\
5 A\end{array}$ & $\begin{array}{c}\text { PZT- } \\
5 H\end{array}$ & $\begin{array}{c}\text { PZT- } \\
81\end{array}$ \\
\hline $\begin{array}{l}\text { Voltage } \\
\begin{array}{l}\text { sensitivity } \\
(\mathrm{V} / \mathrm{MPa})\end{array}\end{array}$ & 16.064 & 16.124 & 17.372 & 16.762 & 16.036 & 14.757 & 11.679 & 16.749 \\
\hline
\end{tabular}

\section{Finite element simulation experiments}

The simulation model of the sensor is established in ANSYS and the grid division of the sensor is completed as shown in figure 3. 


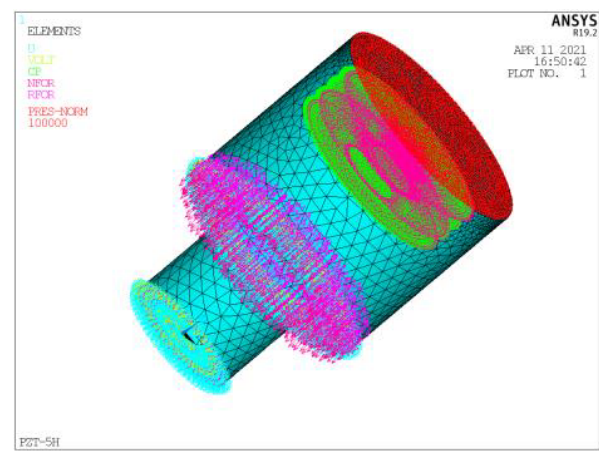

(a) The whole sensor

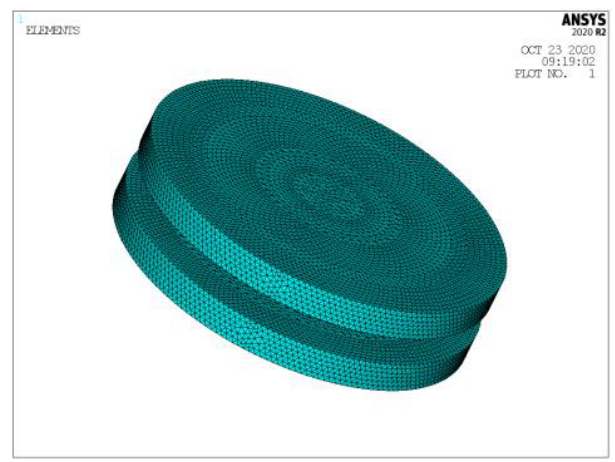

(b) Crystal group

Fig. 3. Meshing model of the sensor.

Selection of materials for components of the sensor, except for force sensitivity, are shown in table 3 .

Table 3. Material model and finite element parameter.

\begin{tabular}{lccccc}
\hline Components & Materials & $\begin{array}{c}\text { Elastic } \\
\text { Modulus }\end{array}$ & $\begin{array}{c}\text { Poisson's } \\
\text { ratio }\end{array}$ & Density & Unit \\
\hline Electrode & $\mathrm{Cu}$ & $1.06 \mathrm{e} 11 \mathrm{~Pa}$ & 0.33 & $8900 \mathrm{~kg} / \mathrm{m} 3$ & Solid186 \\
Plastic ring & PTFE & $2.8 \mathrm{e} 8 \mathrm{~Pa}$ & 0.40 & $2200 \mathrm{~kg} / \mathrm{m} 3$ & Solid186 \\
Diaphragm & 1Cr18Ni9Ti & $2.03 \mathrm{e} 11 \mathrm{~Pa}$ & 0.31 & $7860 \mathrm{~kg} / \mathrm{m} 3$ & Solid186 \\
Lower shell & 1Cr18Ni9Ti & $2.03 \mathrm{e} 11 \mathrm{~Pa}$ & 0.31 & $7860 \mathrm{~kg} / \mathrm{m} 3$ & Solid186 \\
Upper shell & 1Cr18Ni9Ti & $2.03 \mathrm{e} 11 \mathrm{~Pa}$ & 0.31 & $7860 \mathrm{~kg} / \mathrm{m} 3$ & Solid186 \\
Plastic & PTFE & $2.8 \mathrm{e} 8 \mathrm{~Pa}$ & 0.40 & $2200 \mathrm{~kg} / \mathrm{m} 3$ & Solid186 \\
column & & & & \\
\hline
\end{tabular}

There are two kinds of physical fields in piezoelectric sensor [6], stress field and electric field, and they are coupled. Piezoelectric ceramics are anisotropic materials. According to the generalized Hooke's law, the stress field inside the sensor is expressed as following:

$$
x_{i}=\sum_{j=1}^{6} s_{i j} X_{j}, i=1,2,3,4,5,6
$$

where $x_{i}$ is the strain, $X_{j}$ is the stress, and $s_{i j}$ is the elastic compliance coefficient.

According to the dielectric properties of the dielectric material, the expression of the electric field in the sensor is represented as:

$$
D_{m}=\sum_{n=1}^{3} \varepsilon_{m n} E_{n}, m=1,2,3
$$

where $D_{m}$ is electric displacement, $\varepsilon_{m n}$ is dielectric constant, $E_{n}$ is electric field intensity.

The physical phenomenon of force-electricity coupling of the force-sensitive element is represented as:

$$
D_{m}=\sum_{i=1}^{6} d_{m j} X_{j}+\sum_{n=1}^{3} \varepsilon_{m n}^{X} E_{n}
$$




$$
x_{i}=\sum_{j=1}^{6} s_{i j}^{E} X_{j}+\sum_{n=1}^{3} d_{n i} E_{n}
$$

where $d_{m j}$ is piezoelectric strain, the first term of equation (4) is the piezoelectric coupling effect caused by stress, and the second term is the direct polarization effect caused by electric field; the first term of equation (5) is the elastic deformation caused by stress, and the second term is Inverse piezoelectric effect.

Using the materials above as the force-sensitive element, the piezoelectric coupling simulation analysis experiment of the sensor is performed in the ANSYS. 20 to $100 \mathrm{kPa}$ pressure is applied to the sensor in sequence with a step length of $20 \mathrm{kPa}$. The output voltage generated by the force sensor under various pressure loads is obtained through simulation experiments. The simulation diagram shows the corresponding relationship between the output voltage and the pressure load as shown in the figure 4 .

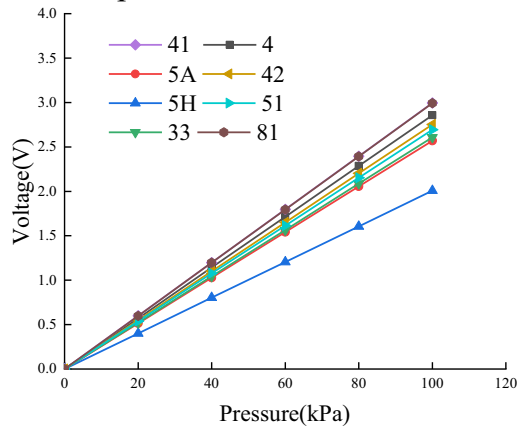

Fig. 4. Relationship between the output voltage of various piezoelectric ceramic crystal groups and the pressure load.

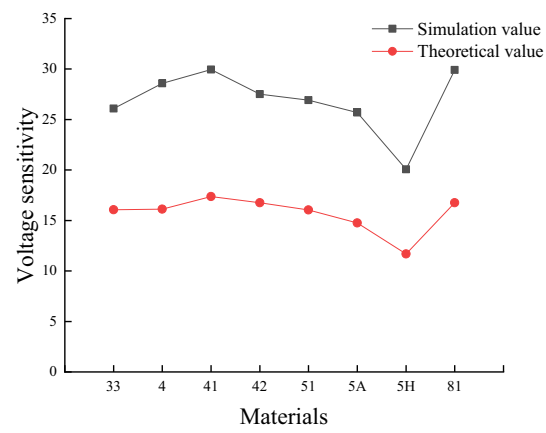

Fig. 5. Comparison of theoretical voltage sensitivity and simulated voltage sensitivity.

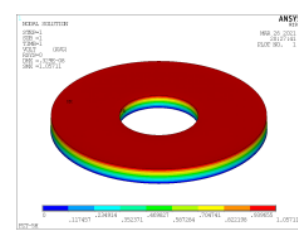

(a) Lower wafer

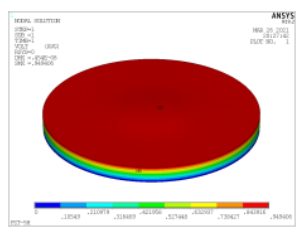

(b) Upper wafer

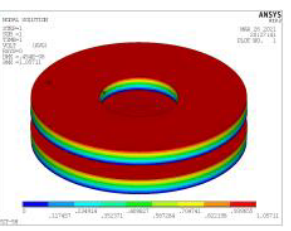

(c) Crystal group

Fig. 6. Simulation diagram of PZT-5H crystal group under 100kPa.

According to figure 5, The change trend of the simulation value is consistent with the theoretical calculation value. Modal analysis experiment of the sensor is carried out in ANSYS, and the first fourth natural frequency of the sensor is obtained as shown in table 4 . 
Figure 7 is the first four simulated natural frequencies of PZT-5H. According to the simulation results, different piezoelectric materials will not affect the natural frequency of the sensor.

Table 4. The first fourth simulated natural frequencies.

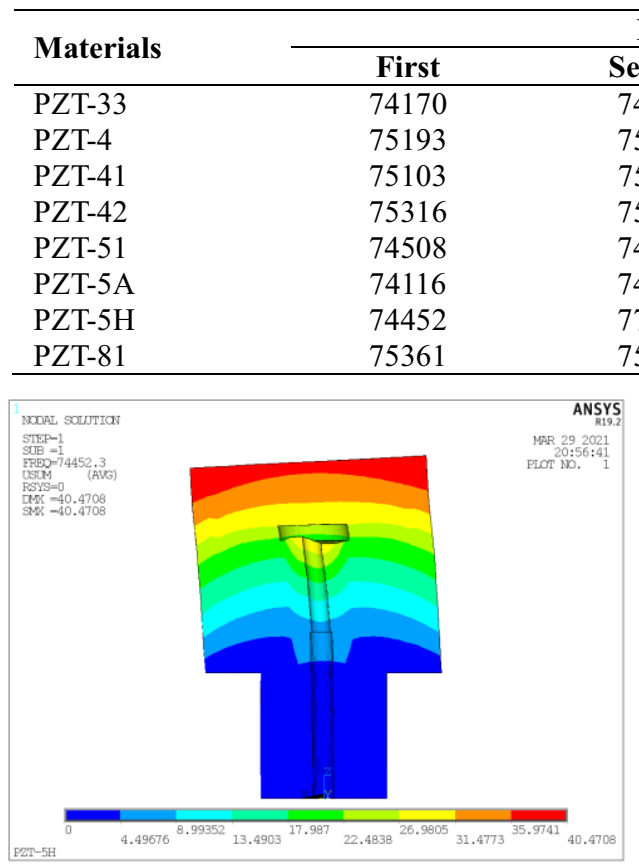

(a) First

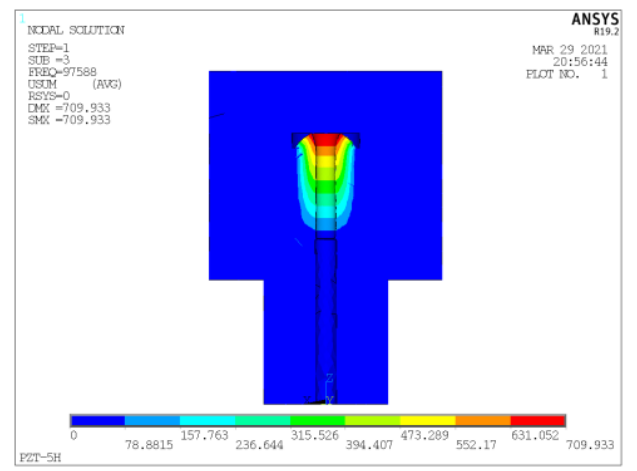

(c) Third

\begin{tabular}{lcc}
\multicolumn{3}{c}{ Natural frequencies } \\
Second & Third & Fourth \\
\hline 74177 & 97587 & 103940 \\
75200 & 97593 & 103940 \\
75110 & 97593 & 103940 \\
75323 & 97594 & 103940 \\
74515 & 97588 & 103940 \\
74122 & 97586 & 103940 \\
77172 & 97588 & 103940 \\
75368 & 97594 & 103940 \\
\hline
\end{tabular}

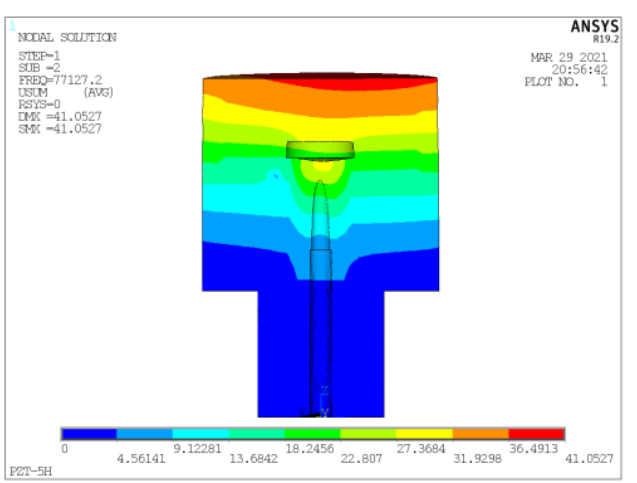

(b) Second

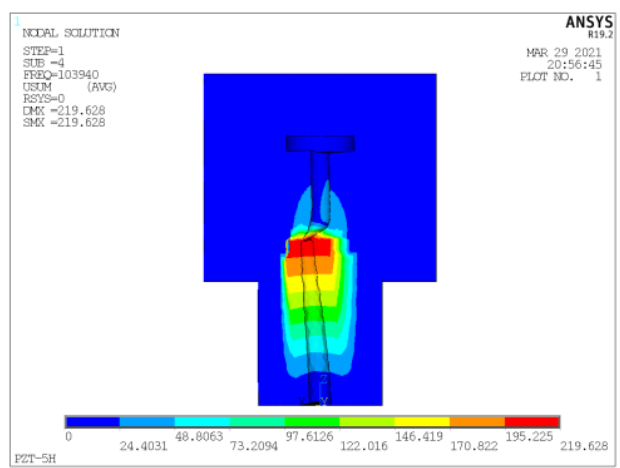

(d) Fourth

Fig. 7. First four simulated natural frequencies of PZT-5H.

\section{Conclusions}

In order to select one kind of appropriate force-sensing element for high-frequency dynamic piezoelectric pressure sensor, this paper performs the piezoelectric coupling simulation analysis experiments in ANSYS multi-physics coupling field. According to the simulation results, the linearity of the output voltage curve of piezoelectric ceramic materials is great. As the force-sensitivity element, $\mathrm{PZT}-5 \mathrm{H}$ is more appropriate than other 
materials. Different piezoelectric materials will not affect the natural frequency of the sensor.

This work is supported by a grant from the National Natural Science Foundation of China (Grant No. 51875250), A Project of Shandong Province Higher Educational Youth Innovation Science and Technology Program (Grant No. 2019KJB018), National Natural Science Foundation of China (Grant No. 51705200)

\section{References}

1. D L Liu and W K Wang 2019 Research status of shock wave overpressure sensor Journal of Telemetry, Tracking and Command vol 40 p 7-15

2. J G Wu and X Y Gao 2018 High temperature piezoelectric materials, devices and Applications Acta Physica Sinica vol 67 p 207701

3. Y J Li and Z K Yang 2020 Research on piezoelectric pressure sensor for shock wave load measurement ISA Transactions vol 104 pp 382-392

4. G C Wang and Y J Li 2021 Acceleration self-compensation mechanism and experimental research on shock wave piezoelectric pressure sensor Mechanical Systems and Signal Processing vol 130 pp 107303

5. M Z Yuan and J R Zhang 2015 Processing method and property study for cementbased piezoelectric composites and sensors Materials Research Innovations vol 19 S1134-S1-138

6. D Y Xu and S F Huang 2014 Electromechanical impedance spectra investigation of impedance-based PZT and cement/polymer based piezoelectric composite sensors Construction \& Building Materials vol 65 p 543-550 